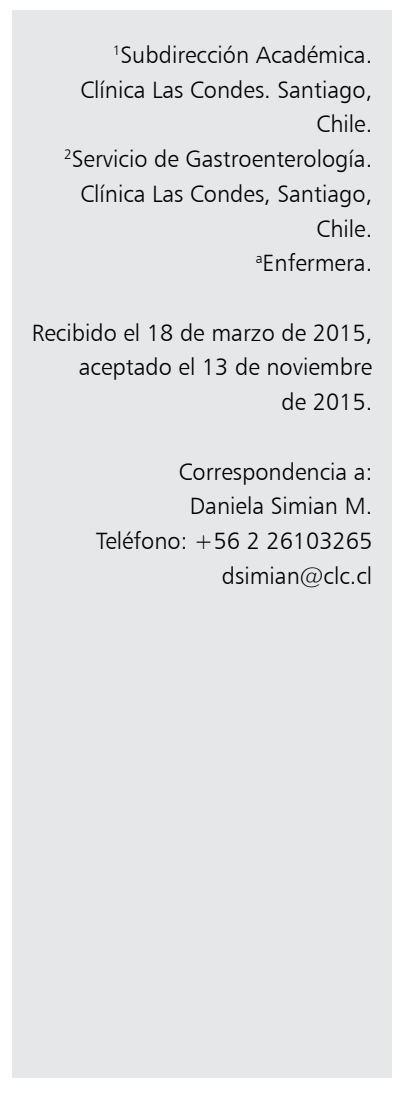

\section{Manejo integral de la enfermedad inflamatoria intestinal: más allá de una terapia farmacológica adecuada}

\author{
DANIELA SIMIAN ${ }^{1, a}$, RODRIGO QUERA ${ }^{2}$
}

\begin{abstract}
Inflammatory Bowel Disease (IBD) is a chronic inflammatory condition of the gastrointestinal tract with medical and psychological complications. Addressing psychosocial aspects of treatment, such as quality of care and disability remains a challenge. The quality of care for IBD patients is not optimal at the present time and there is a variation in the care provided by specialists. Therefore, it is necessary to develop well defined quality indicators to assure the delivery of an adequate care to these patients. The delivery of healthcare for IBD patients is often complex and requires multidisciplinary teams. The ultimate objectives in the treatment of IBD should be to prevent bowel damage, reduce long-term disability and maintain a normal quality of life.
\end{abstract}

(Rev Med Chile 2016; 144: 488-495)

Key words: Colitis, Ulcerative; Crohn Disease; Disability Evaluation; Quality of Health Care; Quality of Life.

\section{工} a enfermedad inflamatoria intestinal (EII) es una enfermedad crónica que incluye dos entidades fundamentales: la enfermedad de Crohn (EC) y la colitis ulcerosa (CU), las cuales se caracterizan por períodos de exacerbación y remisión. A menudo se diagnostican en adultos jóvenes e incluso niños, permaneciendo durante toda la vida del paciente. La etiología de la enfermedad es desconocida y la patogénesis aún no ha sido completamente aclarada. Sin embargo, existe evidencia que ésta se desarrolla por una respuesta inmune descontrolada hacia la microbioma intestinal $\mathrm{u}$ otros factores ambientales, en individuos genéticamente susceptibles. Diferentes estudios han descrito un aumento de la prevalencia e incidencia de la EII, tanto en países industrializados como en vías de desarrollo, reportándose una prevalencia de CU y EC de 505/100.000 y 322/100.000 habitantes en Europa, y de 249/100.000 y 319/100.00 para CU y EC en América del Norte ${ }^{1}$. En Chile no hay estudios de incidencia ni prevalencia, sin em- bargo, nuestro grupo ha sugerido recientemente un aumento de los casos de EC y $\mathrm{CU}^{2}$.

Dentro de los síntomas que refieren los pacientes con EII se encuentran diarrea, hemorragia digestiva baja, dolor abdominal, fiebre, fatiga y baja de peso, entre otros. Pacientes con EC pueden presentar complicaciones como fístulas, abscesos y enfermedad perianal. Manifestaciones extraintestinales como articulares, dermatológicas, oftalmológicas, hepáticas o tromboembólicas se pueden desarrollar tanto en EC como en CU. En relación al tratamiento, existe el riesgo de eventos adversos, destacando los asociados al uso de corticoides $^{3}$. Por último, la necesidad de cirugías y hospitalizaciones están presentes en 10\% y 33\% de los pacientes con CU, y en $47 \%$ y $55 \%$ en $\mathrm{EC}^{2}$.

Hasta hace poco, el control de los síntomas era considerado el objetivo final en el tratamiento de la EII, sin embargo, en los últimos años el concepto de curación de la mucosa ha emergido como un importante objetivo terapéutico ${ }^{4}$. Estudios han 
señalado que la curación de la mucosa con terapia convencional (mesalazina, azatioprina/6-mercaptopurina y metotrexato) y con agentes biológicos tiene el potencial de cambiar la historia natural de la enfermedad, sugiriendo que aquellos que logran esta condición presentan una disminución en la probabilidad de requerir hospitalizaciones y cirugías o desarrollar neoplasia colorrectal ${ }^{4}$.

La elección del tratamiento farmacológico depende de la localización del compromiso inflamatorio, actividad (fase activa o en remisión), severidad, duración de la enfermedad, tolerancia al tratamiento y la presencia de posibles eventos adversos. Además, la elección del tratamiento debe ser definida por la presencia de factores pronósticos como edad joven al diagnóstico, enfermedad extensa, fenotipo penetrante e historia de hábito tabáquico ${ }^{5}$.

Los objetivos del tratamiento de la EII no sólo deben ser reducir la actividad de la enfermedad desde el punto de vista clínico, endoscópico e incluso histológico, sino también mejorar la calidad de vida y el grado de discapacidad de los pacientes, a través de una óptima calidad de atención, idealmente multidisciplinaria (Figura 1).

En este artículo revisaremos los últimos conceptos incorporados en el tratamiento de la EII, los cuales nos parecen relevantes por el rol que cumplen en el manejo y la evolución de esta patología.

\section{Discapacidad}

El término discapacidad constituye un concepto complejo, dinámico y multidimensional que ha sido definido como la limitación crónica que interfiere con la capacidad de dedicarse a las actividades diarias cotidianas, reflejando las limitaciones y restricciones que el paciente tiene en diferentes áreas de su vida, ya sea a nivel laboral, familiar o social ${ }^{6}$.

En estudios internacionales se ha mostrado que pacientes con EII presentan mayor riesgo de discapacidad en comparación con la población general ${ }^{7}$. Esta situación refleja el impacto de la EII sobre la calidad de vida y la productividad de una persona. Estudios han sugerido incluso que la discapacidad puede llegar a ser permanente, dado el compromiso significativo en varios aspectos de la vida de los pacientes ${ }^{8}$. De acuerdo a la definición de discapacidad, ya sea laboral o global, su frecuencia en pacientes con EII ha sido descrita hasta en $34 \%{ }^{9,10}$, siendo más frecuente en pacientes con EC en comparación con el grupo con $\mathrm{CU}^{11}$.

En Chile, según la Encuesta Nacional de Salud 2009-2010, 6,9\% de la población presenta discapacidad en general ${ }^{12}$. Junto con ello, según datos de la Superintendencia de Pensiones de Chile, durante el primer semestre del año 2015, un total de 17.352 personas fueron cubiertas por el Seguro de Invalide ${ }^{13}$. Considerando específicamente

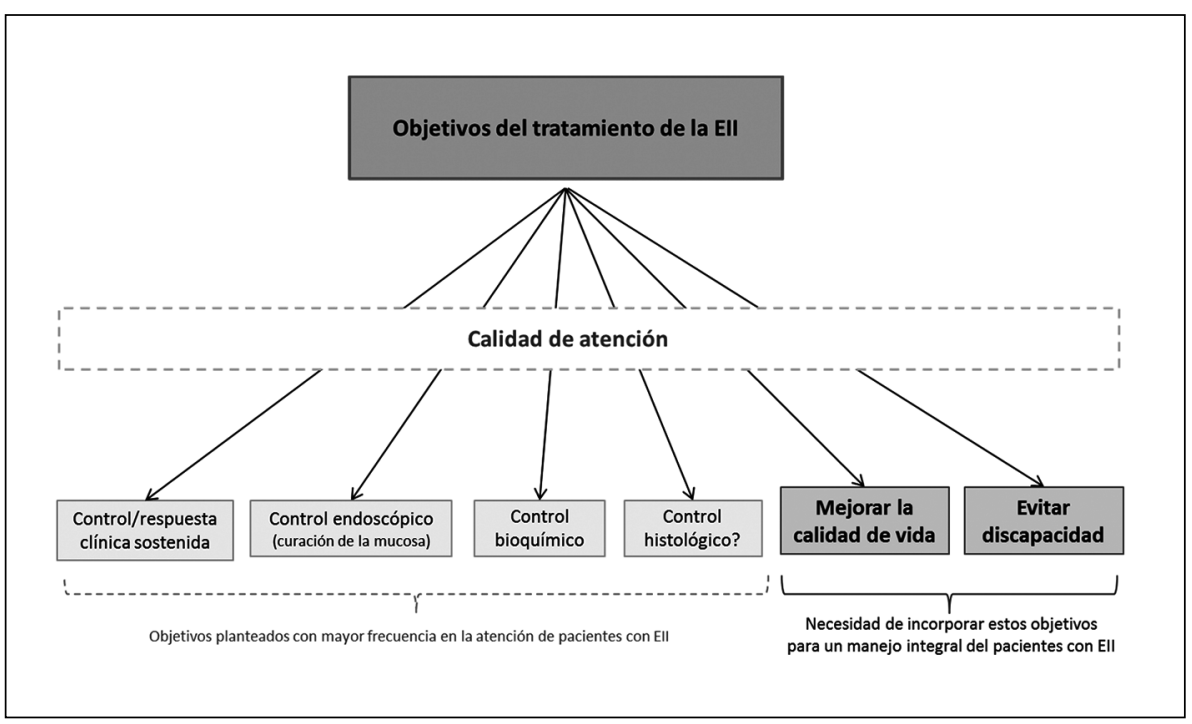

Figura 1. Objetivos del tratamiento de la Ell. Manejo integral. 
discapacidad por alguna patología digestiva, un estudio realizado por el Fondo Nacional de Discapacidad de Chile en el año 2004, estimó que $3,6 \%$ de la población presentaba discapacidad por esta causa ${ }^{14}$.

La mayoría de los estudios realizados en pacientes con EII han analizado la discapacidad laboral o de empleo ${ }^{9,15}$. El estudio realizado por Feagan et al refiere 25\% de discapacidad laboral general, destacando una gran variablilidad dependiendo del país, con un rango desde 20\% en Estados Unidos de Norteamérica hasta 34\% en Europa ${ }^{9}$. Longobardi et al concluyeron que el porcentaje de desempleo en pacientes con EII en Estados Unidos de Norteamérica alcanza 31,5\%, el doble a lo señalado en la población general ${ }^{16}$.

Muy pocos estudios han explorado el impacto de la EII en limitar de manera crónica otros aspectos de la vida diaria, como son la dimensión psicológica y las interacciones sociales ${ }^{11,17}$. Un estudio publicado recientemente ha sugerido que pacientes con EII presentan una alteración en la percepción de su cuerpo, la cual está relacionada con el desarrollo de una discapacidad social y psicológica. El grado de severidad de la EII y el uso de esteroides estarían asociados a la falta de satisfacción corporal en estos pacientes ${ }^{17}$.

En relación a las estrategias terapéuticas, los agentes biológicos serían los únicos fármacos que podrían mejorar el índice de discapacidad en la EII $^{18}$. Por otra parte, el uso de esteroides durante un año ha sido asociado con discapacidad laboral después de 10 años de enfermedad en pacientes con EC y $\mathrm{CU}^{7}$.

Entre los factores ambientales, se ha descrito que el hábito tabáquico exacerba el grado de discapacidad en pacientes con EC, no así en el grupo con $\mathrm{CU}^{18}$, lo que se podría explicar por su asociación a un curso más severo, mayor frecuencia de recaídas y recurrencia post-quirúrgica en pacientes con EC.

Otros factores, como enfermedad activa de larga data, mayor grado de estrés psicológico, mayor nivel de percepción de estrés y una pobre calidad de vida se han asociado a discapacidad en estos pacientes ${ }^{11}$. Otros han señalado que factores como sexo femenino, menor nivel de educación, dolor de columna, más de dos cirugías, más de dos hospitalizaciones por crisis también estarían relacionados con un mayor riesgo de discapacidad $^{9,15}$. Sin duda, la falta de una herramienta es- tandarizada de medición para cuantificar el grado de discapacidad hace difícil la comparación de los resultados entre diferentes estudios ${ }^{10}$.

Peyrin-Biroulet et al han desarrollado recientemente un índice de discapacidad para la EII, que permite evaluar de manera objetiva y adecuada las limitaciones funcionales en estos pacientes ${ }^{19}$. Este índice ha demostrado ser confiable, reproducible y sensible en la capacidad de detectar discapaci$\mathrm{dad}^{18}$. Es posible que en un futuro cercano, esta herramienta sea útil en la práctica clínica, en el planteamiento de estudios y en la evaluación de la atención de salud en la EII.

\section{Calidad de vida}

La calidad de vida relacionada con salud ha sido definida como el nivel de bienestar derivado de la evaluación que la persona realiza de diversos dominios de su vida, considerando el impacto que en éstos tiene su estado de salud, teniendo por lo tanto un valor subjetivo ${ }^{20}$.

Estudios han reportado que la calidad de vida en pacientes con enfermedades crónicas se ve afectada $^{21,22}$, lo que incluye patologías digestivas como síndrome intestino irritable (SII), enfermedad celíaca y EII. En el caso del SII, diferentes instrumentos han sido utilizados para evaluar el impacto de esta patología en la calidad de vida, destacando el IBSQOL, IBS-QOL, IBS-36 y el QLICD $^{23-26}$. Un estudio que utilizó el cuestionario SF-36 demostró que pacientes con SII presentaban un mayor deterioro de calidad de vida comparado con pacientes con reflujo gastroesofágico, diabetes mellitus o enfermedad renal crónica ${ }^{24}$. Pace y cols, utilizando el mismo instrumento, demostraron que pacientes con SII atendidos en un centro de referencia podrían tener un deterioro de su calidad de vida similar a la de los pacientes con $\mathrm{EII}^{25}$. Sin embargo, diferentes variables deben ser consideradas al momento de determinar los factores que podrían influir en el deterioro de la calidad de vida, como es el caso de la edad, género, severidad de los síntomas, tratamiento y subtipo de $\mathrm{SII}^{27}$. En el caso de la enfermedad celíaca, diferentes instrumentos, como el SF-36, CDQ y CD-QOL, han sido utilizados para evaluar calidad de vida ${ }^{28,29}$. Un estudio que incluyó pacientes con enfermedad celíaca y EII demostró que aquellos que presentaban síntomas compatibles con reflujo gastroesofágico y SII tenían un mayor deterioro 
de su calidad de vida comparada con aquellos que no presentaban estas dos patologías $(\mathrm{p}<0,0001)$, sugiriendo que una adecuada evaluación y manejo debe ser realizada con el fin de descartar la presencia de otras patologías digestivas que puedan alterar su calidad de vida ${ }^{30}$.

En el caso de la EII, la calidad de vida se ve afectada significativamente, en especial durante los períodos de crisis, aumentando su compromiso de acuerdo a la severidad de la enfermedad ${ }^{8}$.

El deterioro de la calidad de vida de los pacientes con EII es consecuencia de la cronicidad de la enfermedad, la imposibilidad de predecir su evolución, la severidad de sus crisis, la necesidad de hospitalizaciones y cirugía y la posibilidad de eventos adversos a los fármacos utilizados para su tratamiento. El hecho que la enfermedad tenga períodos de remisión y actividad genera estrés en el paciente, lo que compromete la adherencia al tratamiento y a las recomendaciones dadas por el equipo de salud, aumentando en consecuencia el riesgo de recaída y empeorando la calidad de vida ${ }^{19}$. Pacientes con EII presentan un mayor grado de preocupación por las complicaciones de la enfermedad y riesgo de depresión, en comparación con otras enfermedades crónicas como artritis reumatoidea, asma o migraña ${ }^{21}$.

Diversos estudios han descrito factores que estarían asociados a un deterioro en la calidad de vida de los pacientes con EII. Dentro de ellos destacan pacientes mujeres, que presenten un mayor grado de estrés, menor apoyo social, un mayor número de recaídas de su enfermedad, síntomas persistentes después de 10 años de seguimiento y una pensión de invalidez por $\mathrm{EII}^{31,32}$. La EC es una enfermedad heterogénea que puede presentar tres fenotipos distintos (inflamatorio, penetrante y fistulizante), los cuales resultan de una compleja interacción entre factores genéticos, inmunológicos y ambientales. Recientemente, un estudio que incluyó 361 pacientes con EC sugiere que aquellos pacientes con un retraso en el diagnóstico presentan una peor calidad de vida $^{33}$. Esta asociación puede ser explicada por un mayor fracaso al tratamiento médico y necesidad de cirugía en pacientes con diagnóstico tardío y una mayor frustración debido a síntomas no controlados por sí mismos.

El déficit de vitamina $\mathrm{D}$ es frecuente en pacientes con EII, incluso en aquellos diagnosticados recientemente ${ }^{34}$. Bajas concentraciones de esta vitamina estarían asociadas a un aumento en la actividad de la CU y EC ${ }^{34}$. Recientemente, Hlavaty et al. han sugerido que concentraciones disminuidas de vitamina $\mathrm{D}$ estarían asociadas a una baja calidad de vida en pacientes con EII. La suplementación de vitamina $\mathrm{D}$ hasta alcanzar concentraciones séricas de 50-59 ng/ml permitiría mejorar su calidad de vida ${ }^{35}$.

En relación al tratamiento, infliximab, un seguro y efectivo agente biológico para el tratamiento de la EII, ha demostrado mejorar de manera significativa la calidad de vida al compararlo con el grupo tratado con terapia convencional ${ }^{36}$. Esto se podría explicar por la remisión clínica continua y la curación de la mucosa cuando se utiliza esta terapia.

La medición de la calidad de vida no sólo es importante en la evaluación clínica, sino también en el manejo terapéutico del paciente. El equipo médico tratante debe ser capaz de identificar las necesidades del paciente, tratar de encontrar las mejores opciones para ellos y planificar un cuidado de su estado de salud, de tal manera que la calidad de vida de ellos sea mejor. Los instrumentos para medir calidad de vida relacionada a la salud, entregan una importante información acerca de la percepción de los pacientes sobre su enfermedad y cómo las estrategias terapéuticas utilizadas la afectan. Para medir la calidad de vida en la práctica clínica es importante que estos instrumentos sean breves, efectivos, validados, confiables y aplicables a todos los pacientes con EII. Sin embargo, la mayoría de las herramientas utilizadas en la actualidad son largas y consumen tiempo, lo que explica su bajo uso en la práctica clínica. Los instrumentos clásicos para medir calidad de vida son el SF-36 y el SF-12, los cuales se han utilizado en algunos estudios en $\mathrm{EII}^{32,36,37}$, pero no son específicos para esta patología. El instrumento más comúnmente utilizado en la práctica clínica $y$ en los estudios es el IBDQ ${ }^{31,32-36,38}$ desarrollado en la Universidad de McMaster, Ontario, Canadá, el cual consta de 32 preguntas $^{39}$. Este cuestionario se encuentra traducido y validado al español ${ }^{38}$. Recientemente, Alrubaiy et al desarrollaron un nuevo cuestionario (CUCQ-8) que aplicaron en 205 pacientes con EII, demostrando que podría ser un método más corto y eficiente para evaluar la calidad de vida en pacientes con $\mathrm{EII}^{40}$, el cual debe ser utilizado por otros grupos para poder validar su uso en la práctica. 
Tabla 1. Factores que podrían influir en el grado de discapacidad y en la calidad de vida de los pacientes con Ell

\begin{tabular}{|c|c|c|}
\hline Factor & Grado de discapacidad & Calidad de vida \\
\hline Enfermedad de Crohn ${ }^{11}$ & $\checkmark$ & \\
\hline Grado de severidad de la EII ${ }^{8,17}$ & $\checkmark$ & $\checkmark$ \\
\hline Uso de esteroides ${ }^{7,17}$ & $\checkmark$ & \\
\hline Hábito tabáquico en $\mathrm{EC}^{18}$ & $\checkmark$ & \\
\hline Enfermedad activa de larga data (mayor 10 años de evolución) 11,31,32 & $\checkmark$ & $\checkmark$ \\
\hline Mayor grado de estrés psicológico ${ }^{11,31,32}$ & $\checkmark$ & $\checkmark$ \\
\hline Pobre calidad de vida ${ }^{11}$ & $\checkmark$ & \\
\hline Género femenino $9,15,31,32$ & $\checkmark$ & $\checkmark$ \\
\hline Menor nivel de educación $n^{9,15}$ & $\checkmark$ & \\
\hline Más de dos cirugías por El|9,15 & $\checkmark$ & \\
\hline Más de dos hospitalizaciones por crisis $^{31,32}$ & $\checkmark$ & \\
\hline Menor apoyo social ${ }^{31,32}$ & & $\checkmark$ \\
\hline Mayor número de recaídas de su enfermedad ${ }^{31,32}$ & & $\checkmark$ \\
\hline Pensión de invalidez por El|21,32 & & $\checkmark$ \\
\hline Retraso en el diagnóstico de la $\mathrm{EC}^{33}$ & & $\checkmark$ \\
\hline Déficit de vitamina $D^{35}$ & & $\checkmark$ \\
\hline
\end{tabular}

El gastroenterólogo no sólo debe preocuparse del tratamiento médico de la EII, sino que también lograr un diálogo con el paciente en relación a sus expectativas, factores estresantes, las consecuencias que puedan traer los períodos de crisis desde el punto de vista psicológico y social. Así podrán determinar en conjunto qué aspectos ayudarían a disminuir el grado de discapacidad y con ello mejorar su calidad de vida ${ }^{31,41}$. En la Tabla 1 se muestran los factores que podrían afectar estas dos variables.

\section{Calidad de la atención}

En la actualidad, el tratamiento de cualquier enfermedad crónica debería estar enfocado en entregar una atención continua y de calidad, con el fin de disminuir su morbilidad y mortalidad. Asch et al han señalado que sólo $57 \%$ de los pacientes que se atienden regularmente en una consulta reciben una atención de calidad enfocada a disminuir complicaciones, hospitalizaciones o el riesgo de muerte $^{42}$. Además, 53\% de los pacientes con EII señalan sentirse incapaces de decir alguna cosa importante relacionada a su salud en la consulta con su equipo tratante ${ }^{43}$.

Actualmente, la calidad de la atención de los pacientes con EII no es óptima y por lo tanto necesita ser mejorada. Reddy et al. han sugerido que pacientes con EII a menudo no reciben un tratamiento adecuado, lo que se traduce en dosis subóptimas de mesalazina e inmunosupresores, prolongado uso de esteroides y suplementos de calcio y vitamina $D$, inadecuada vigilancia de cáncer colorrectal y ausencia de indicación de vacunas $^{44}$. Por otra parte, la percepción de los pacientes con EII sobre la calidad de la atención que ellos tienen está determinada por la presencia de factores pronósticos y por el tipo de terapia que reciben para el manejo de su enfermedad ${ }^{45}$.

La entrega de atención de salud a pacientes con EII es en general compleja y requiere la coordinación entre gastroenterólogos y coloproctólogos, preferentemente con experiencia en EII, radiólogos e idealmente enfermeras especializadas 
en EII. La mejor atención de pacientes con EII parece provenir de un manejo multidisciplinario, específicamente de centros especializados en esta patología ${ }^{46}$. A pesar de la disponibilidad de guías sobre el manejo de la $\mathrm{EII}^{5}$, existe evidencia que sugiere diferencias en la atención entregada a pacientes con EII por parte de equipos de salud con experiencia en esta patología y equipos de atención primaria ${ }^{47}$. Estas diferencias se darían incluso entre equipos con experiencia en el manejo de pacientes con EII, como es el caso de definir el inicio del tratamiento farmacológico en pacientes con diagnóstico reciente de $\mathrm{EC}^{47}$. Esta variabilidad en la atención es considerada un índice de calidad de atención deficiente.

Con el fin de mejorar la atención de salud en pacientes con EII, debemos enfocarnos en realizar cambios que incluyan la creación de unidades para pacientes con EII, tener la disposición a entregar un sistema de atención continua, estandarizar los cuidados de atención y realizar una educación a los equipos de salud primaria sobre temas relacionados con el manejo de los pacientes con EII. Recientemente, un estudio concluye que un servicio de EII ideal debe ser multidisciplinario, integrando en éste el apoyo de enfermeras, nutricionistas/nutriólogos y psicólogos ${ }^{48}$. Por otra parte, cuestionarios de evaluación e índices de medición de la calidad de atención en EII han sido sugeridos por diferentes organismos ${ }^{49}$. En un estudio realizado en Europa, en 25 países, que incluyó 4.670 pacientes, $54 \%$ de ellos refirió no tener la oportunidad de contarle al médico algo potencialmente importante acerca de su enfermedad, y por otro lado, $64 \%$ siente que el gastroenterólogo debería haber hecho más preguntas de profundización para entender mejor su estado de salud actual ${ }^{41}$. Sin duda, la implementación de estas medidas tendrá un significativo impacto en el manejo de los pacientes con EII. Estudios han demostrado que un modelo de atención integrado puede mejorar los resultados clínicos y psicosociales de estos pacientes, siendo incluso costo-efectivo $^{50}$.

Además, está claro que la satisfacción por parte del paciente es un componente importante de la calidad de la atención. Esta afirmación se basa en que el grado de concordancia entre las expectativas y necesidades del paciente y la atención entregada por equipo tratante afecta la adherencia al tratamiento.

\section{Conclusión}

Existe la necesidad de considerar la EII como una enfermedad progresiva y entender la importancia de un diagnóstico precoz con una intervención multidisciplinaria en estadios iniciales de la enfermedad. Sin duda, fomentar la colaboración entre el paciente y el equipo tratante permitirá optimizar la adherencia a los controles y a la terapia. La motivación de comenzar y mantener el tratamiento farmacológico, más allá del riesgo de los eventos adversos, permitirá alcanzar no sólo los objetivos de remisión clínica sostenida, sino también prevenir la discapacidad y mejorar la calidad de vida de nuestros pacientes.

\section{Referencias}

1. Molodecky NA, Soon IS, Rabi DM, Ghali WA, Ferris M, Chernoff G, et al. Increasing incidence and prevalence of the inflammatory bowel diseases with time, based on systematic review. Gastroenterology 2012; 142: 46-54.

2. Simian D, Estay C, Lubascher J, Acuña R, Kronberg U, Figueroa C, et al. Enfermedad inflamatoria intestinal a partir de una experiencia local. Rev Med Chile 2014; 142: 1006-13.

3. Meyer L, Simian D, Lubascher J, Acuña R, Figueroa C, Silva G, et al. Eventos adversos en la terapia farmacológica de la enfermedad inflamatoria intestinal. Rev Med Chile 2015; 143: 7-13.

4. Florholmen J. Mucosal healing in the era of biologic agents in treatment of inflammatory bowel disease. Scand J Gastroenterol 2015; 50: 43-52.

5. Mowat C, Cole A, Windsod A, Ahmad T, Arnott I, Driscoll R, et al. Guidelines for the management of inflammatory bowel disease in adults. Gut 2011; 60: 571-607.

6. World Health Organization. International Classification of Functioning, Disability and Health (ICF). Geneva: WHO, 2001. Disponible en: http://www.who.int/classifications/icf/en [Consultado el 23 de febrero de 2015].

7. Høivik ML, Moum B, Solberg IC, Henriksen M, Cvancarova M, Bernklev T, et al. Work disability in inflammatory bowel disease patients 10 years after disease onset: results from the IBSEN Study. Gut 2013; 62: 368-75.

8. Vidal A, Gómez-Gil E, Sans M, Portella MJ, Salamero M, Piqué JM, et al. Health-related quality of life in inflammatory bowel disease patients: the role of psychopathology and personality. Inflamm Bowel Dis 2008; 14: 977-83.

9. Feagan BG, Bala M, Yan S, Olson A, Hanauer S. Unemployment and disability in patients with moderately to 
severely active Crohn's disease. J Clin Gastroenterol 2005; 39: 390-5.

10. Büsch K, Sonnenberg A, Bansback N. Impact of inflammatory bowel disease on disability. Curr Gastroenterol Rep 2014; 16: 414.

11. Israeli E, Graff LA, Clara I, Walker JR, Lix LM, Targownik LE, et al. Low prevalence of disability among patients with inflammatory bowel diseases a decade after diagnosis. Clin Gastroenterol Hepatol 2014; 12: 1330-7.

12. Ministerio de Salud, Gobierno de Chile. Encuesta Nacional de Salud ENS Chile 2009-2010. Disponible en: http://web.minsal.cl/portal/url/item/bcb03d7bc28b64dfe040010165012d23.pdf. [Consultado el 21 de octubre de 2015].

13. Superintendencia de Pensiones de Chile. Disponible en: http://www.spensiones.cl/safpstats/stats/.sc.php?_ci$\mathrm{d}=46$ inval. [Consultado el 25 de octubre de 2015].

14. Fondo Nacional de la Discapacidad. First National Study on Disability. ENDISC-CIF, Chile 2004. Disponible en: http://www.ine.cl/canales/chile_estadistico/encuestas_discapacidad/pdf/estudionacionaldeladiscapacidad(ingles).pdf [Consultado el 20 de febrero de 2015].

15. van der Valk ME, Mangen MJ, Leenders M, Dijkstra G, van Bodegraven AA, Fidder HH, et al. Risks factors of work disability in patients with inflammatory bowel disease-a Dutch nationwide web-based survey: work disability in inflammatory bowel disease. J Crohns Colitis 2014; 8: 590-7.

16. Longobardi T1, Jacobs P, Bernstein CN. Work losses related to inflammatory bowel disease in the United States: results from the National Health Interview Survey. Am J Gastroenterol 2003; 98: 1064-72.

17. McDermott E, Mullen G, Moloney J, Keegan D, Bryne $\mathrm{K}$, Doherty GA, et al. Body image dissatisfaction: clinical features, and psychosocial disability in inflammatory bowel disease. Inflamm Bowel Dis 2015; 21: 353-60.

18. Leong R, Huang T, Ko Y, Jeon A, Chang J, Kohler F, et al. Prospective validation study of the International Classification of Functioning, Disability and Health score in Crohn's disease and ulcerative colitis. J Crohns Colitis 2014; 8: 1237-45.

19. Peyrin-Biroulet L, Cieza A, Sandborn W, Coenen M, Chowers Y, Hibi T, et al. Development of the first disability index for inflammatory bowel disease based on the international classification of functioning, disability and health. Gut 2012; 61: 241-7.

20. Urzúa MA. Calidad de vida relacionada con la salud: Elementos conceptuales. Rev Med Chile 2010; 138: 35865.

21. Rubin DT, Dubinsky MC, Panaccione R, Siegel CA, Binion DG, Kane SV, et al. The impact of ulcerative colitis on patients' lives compared to other chronic diseases: a patient survey. Dig Dis Sci 2010; 55: 1044-52.

22. Arne M, Janson C, Janson S, Boman G, Lindqvist U, Berne C, et al. Physical activity and quality of life in subjects with chronic disease: chronic obstructive pulmonary disease compared with rheumatoid arthritis and diabetes mellitus. Scand J Prim Health Care 2009; 27: 141-7.

23. Groll D, Vanner SJ, Depew WT, DaCosta LR, Simon JB, Groll A, et al. The IBS-36: a new quality of life measure for irritable bowel syndrome. Am J Gastroenterol 2002; 97: 962-71.

24. Gralnek IM, Hays RD, Kilbourne A, Naliboff B, Mayer EA. The impact of irritable bowel syndrome on health-related quality of life. Gastroenterology 2000; 119: 654-60.

25. Pace F, Molteni P, Bollani S, Sarzi-Puttini P, Stockbrügger R, Bianchi Porro G, et al. Inflammatory bowel disease versus irritable bowel syndrome: a hospital-based, case-control study of disease impact on quality of life. Scand J Gastroenterol 2003; 38: 1031-8.

26. Lei P, Lei G, Tian J, Zhou Z, Zhao M, Wan C. Development and validation of the irritable bowel syndrome scale under the system of quality of life instruments for chronic diseases QLICD-IBS: combinations of classical test theory and generalizability theory. Int J Colorectal Dis 2014; 29: 1245-55.

27. Mönnikes H. Quality of life in patients with irritable bowel syndrome. J Clin Gastroenterol 2011; 45 Suppl: S98-101.

28. Häuser W, Gold J, Stallmach A, Caspary WF, Stein J. Development and validation of the Celiac Disease Questionnaire (CDQ), a disease-specific health-related quality of life measure for adult patients with celiac disease. J Clin Gastroenterol 2007; 41: 157-66.

29. Dorn SD, Hernández L, Minaya MT, Morris CB, Hu Y, Leserman J, et al. The development and validation of a new coeliac disease quality of life survey (CD-QOL). Aliment Pharmacol Ther 2010; 31: 666-75.

30. Barratt SM, Leeds JS, Robinson K, Shah PJ, Lobo AJ, McAlindon ME, et al. Reflux and irritable bowel syndrome are negative predictors of quality of life in coeliac disease and inflammatory bowel disease. Eur J Gastroenterol Hepatol 2011; 23: 159-65.

31. Tabibian A, Tabibian JH, Beckmean L, Raffals LL, Papadakis K, Kane S. Predictors of health-related quality of life and adherence in Crohn's disease and ulcerative colitis: Implications for clinical management. Dig Dis Sci 2015; 60: 1366-74.

32. Huppertz-Hauss G, Høivik ML, Langholz E, Odes S, Småstuen $\mathrm{M}$, Stockbrugger $\mathrm{R}$, et al. Health-related 
quality of life in inflammatory bowel disease in a European-wide population-based cohort 10 years after diagnosis. Inflamm Bowel Dis 2015; 21: 337-44.

33. Pellino G, Sciaudone G, Selvaggi F, Riegler G. Delayed diagnosis in influenced by the clinical pattern of Cro$\mathrm{hn}$ 's disease and effects treatment outcomes and quality of life in the long term: a cross-sectional study of 361 patients in Southern Italy. Eur J Gastroenterol 2015; 27: 175-81.

34. Ulitsky A, Ananthakrishann AN, Naik A, Skaros S, Zadvornova Y, Binion DG, et al. Vitamin D deficiency in patients with inflammatory bowel disease: association with disease activity and quality of life. JPEN J Parenter Enteral Nutr 2011; 35: 308-16.

35. Hlavaty A, Krajcovicova A, Koller T, Toth J, Nevidanska M, Huorka M, et al. Higher vitamin D serum concentration increase health quality of life in patients with inflammatory bowel diseases. World J Gastroenterol 2014; 20: 15787-96.

36. Xu J, Lin H, Feng X, Tang M, Shen J, Ran Z. Different therapeutic approaches on quality of life in patients with inflammatory bowel disease. BMC Gastroenterol 2014; 14: 199.

37. Hoivik ML, Moum B, Solberg IC, Cvancarova M, Hoie $\mathrm{O}$, Vatn $\mathrm{MH}$, et al. Health-related quality of life in patients with ulcerative colitis after a 10-year disease course: results from the IBSEN study. Inflamm Bowel Dis 2012; 18: 1540-9.

38. Masachs M1, Casellas F, Malagelada JR. Traducción, adaptación y validación al español del cuestionario de calidad de vida de 32 ítems (IBDQ-32) de la enfermedad inflamatoria intestinal. Rev Esp Enferm Dig 2007; 99: 511-9.

39. Guyatt G, Mitchell A, Irvine EJ, Singer J, Williams N, Goodacre R, et al. A new measure of health status for clinical trials in inflammatory bowel disease. Gastroenterology 1989; 96: 804-10.

40. Alrubaiy L, Cheung WY, Dodds P, Hutchings HA, Russell IT, Watkins A, et al. Development of a short questionnaire to assess the quality of life in Crohn's disease and ulcerative colitis. J Crohns Colitis 2015; 9: 66-76.

41. Lönnfors S, Vermeire S, Greco M, Hommes D, Bell C, Avedano L. IBD and health-related quality of life-discovering the true impact. J Crohns Colitis 2014; 8: 1281-6.
42. Asch SM, McGlynn EA, Hogan MM, Hayward RA, Shekelle P, Rubenstein L, et al. Comparison of quality of care for patients in the Veterans Health Administration and patients in a rational sample. Ann Intern Med 2004; 141: 938-45.

43. Wilson B, Lönnfors S, Hommes DW, Vermeire S, Greco M, Bell C, et al. A European Crohn's and ulcerative colitis patient life IMPACT survey. Wight Congress of the European Crohn's and Colitis Organization; 2013. Abstract P406.Diponible en: https://www.ecco-ibd.eu/index. php/publications/congress-abstract-s/abstracts-2012/ item/p406-a-euro.html. [Consultado el 20 de octubre de 2015].

44. Reddy SI, Friedman S, Telford JJ, Strate L, Ookudo R, Banks PA. Are patients with inflammatory bowel disease receiving optimal care? Am J Gastroenterology 2005; 100: 1357-61.

45. Vasudevan A, Arachchi A, van Langenberg DR. Assessing patient satisfaction in inflammatory bowel disease using the QUOTE-IBD survey: A small step for clinicians, a potentially large step for improving quality of care. J Crohns Colitis 2013; 7: e367-74.

46. Ricci C, Lanzarotto F, Lanzini A. The multidisciplinary team for management of inflammatory bowel diseases. Dig Liver Dis 2008; 40 (Suppl 2): S285-8.

47. Esrailian E, Spiegel BM, Targownik LE, Dubinsky MC, Targan SR, Gralnek IM. Differences in the management of Crohn's disease among experts and community providers based on a national survey of sample cases vignettes. Aliment Pharmacol Ther 2007; 26: 1005-18.

48. Mikocka-Walus A, Andrews JM, Rampton D, Goodhand J, van der Woude J, Bernstein CN. How can we improve models of care in inflammatory bowel disease? An international survey of IBD health professionals. J Crohns Colitis 2014; 8: 1668-74.

49. Melmed GY, Siegel CA, Spiegel BM, Allen JI, Cima R, Colombel JF, et al. Quality indicators for inflammatory Bowel disease: development of process and outcomes measures. Inflamm Bowel Dis 2013; 19: 662-8.

50. Sack C, Phan VA, Grafton R, Holtmann G, van Langenberg DR, Brett K, et al. A chronic care model significantly decreases costs and healthcare utilization in patients with inflammatory bowel disease. J Crohns Colitis 2012; 6: 302-10. 